\title{
The MAGIC of CINEMA: first in-flight science results from a miniaturised anisotropic magnetoresistive magnetometer
}

\author{
M. O. Archer ${ }^{1, *}$, T. S. Horbury ${ }^{1}$, P. Brown ${ }^{1}$, J. P. Eastwood ${ }^{1}$, T. M. Oddy ${ }^{1}$, B. J. Whiteside ${ }^{1}$, and J. G. Sample ${ }^{2}$ \\ ${ }^{1}$ Space and Atmospheric Physics, The Blackett Laboratory, Imperial College London, London, SW7 2AZ, UK \\ ${ }^{2}$ Space Sciences Laboratory, University of California Berkeley, 7 Gauss Way, Berkeley, CA 94720, USA \\ * now at: School of Physics \& Astronomy, Queen Mary University of London, London, E1 4NS, UK
}

Correspondence to: M. O. Archer (m.archer10@imperial.ac.uk)

Received: 27 February 2015 - Revised: 16 May 2015 - Accepted: 20 May 2015 - Published: 12 June 2015

\begin{abstract}
We present the first in-flight results from a novel miniaturised anisotropic magnetoresistive space magnetometer, MAGIC (MAGnetometer from Imperial College), aboard the first CINEMA (CubeSat for Ions, Neutrals, Electrons and MAgnetic fields) spacecraft in low Earth orbit. An attitudeindependent calibration technique is detailed using the International Geomagnetic Reference Field (IGRF), which is temperature dependent in the case of the outboard sensor. We show that the sensors accurately measure the expected absolute field to within $2 \%$ in attitude mode and $1 \%$ in science mode. Using a simple method we are able to estimate the spacecraft's attitude using the magnetometer only, thus characterising CINEMA's spin, precession and nutation. Finally, we show that the outboard sensor is capable of detecting transient physical signals with amplitudes of $\sim 20-60 \mathrm{nT}$. These include field-aligned currents at the auroral oval, qualitatively similar to previous observations, which agree in location with measurements from the DMSP (Defense Meteorological Satellite Program) and POES (Polar-orbiting Operational Environmental Satellites) spacecraft. Thus, we demonstrate and discuss the potential science capabilities of the MAGIC instrument onboard a CubeSat platform.
\end{abstract}

Keywords. Magnetospheric physics (current systems; instruments and techniques) - Ionosphere (instruments and techniques)

\section{Introduction}

Data from magnetometers on spacecraft are typically used for one, or both, of two purposes: for the determination of the spacecraft attitude and for the measurement of physical pro- cesses local to, or indeed far from, the spacecraft. No measurement is perfect, and the measurement of magnetic fields is particularly challenging given their low values and the particularly small nature of the variations that must be detected for some applications; see, e.g., Acuña (2002) for a historical description of space magnetometer techniques. All sensor and spacecraft environments have different capabilities, and every application of magnetometer data has different requirements in terms of cadence, accuracy, noise, etc.; thus, the intended use cannot be isolated from the methods used to recover accurate magnetic field measurements since one drives the other.

Attitude control knowledge often results in rather coarse requirements of just a few degrees (e.g. Natanson et al., 1990), corresponding to an absolute accuracy in a given field component of $\sim 2000 \mathrm{nT}$ or greater at low Earth orbit (LEO), equivalent to at least $\sim 4 \%$. In contrast, for scientific applications the requirements are more stringent and depend on the precise goal: for example the ESA Swarm mission aims for sub-nT absolute precision (Friis-Christensen et al., 2006). However, if the scientific requirement is to be able to detect transient signals in magnetometer data at LEO, such as field-aligned currents at the auroral oval (e.g. the review of Baumjohann, 1982), then such absolute precision in the overall magnetic field is not required. It is therefore important to assess what it is possible to achieve with a magnetometer, given the quality of the sensor and the environment it is in.

CubeSats offer the possibility of low-cost spacecraft in orbit around the Earth equipped with scientific instruments, e.g. for space weather monitoring purposes (cf. Li et al., 2013). The CubeSat specification, however, constrains both dimensions (a three-unit CubeSat is $10 \mathrm{~cm} \times 10 \mathrm{~cm} \times 30 \mathrm{~cm}$ with 
Table 1. Summary of the MAGIC data used in this paper including the orbital elements of CINEMA, MAGIC modes and geomagnetic indices.

\begin{tabular}{lcc}
\hline & 27 Sep 2012 & 19 Nov 2013 \\
\hline Perigee altitude $(\mathrm{km})$ & 478 & 495 \\
Apogee altitude $(\mathrm{km})$ & 786 & 751 \\
Inclination $\left({ }^{\circ}\right)$ & 64.68 & 64.67 \\
Period (min) & 97.35 & 97.18 \\
$\Delta t_{\mathrm{TLE}}(\mathrm{h})$ & 27 & 37 \\
\hline MAGIC mode & Attitude* & Science \\
Sensor & IB & OB \\
Duration (min) & 231 & 46 \\
Cadence (s) & $10-16$ & 0.128 \\
\hline Kp & 1.2 & 1.0 \\
Dst (nT) & -6 & 8 \\
AE (nT) & 48 & 31 \\
\hline
\end{tabular}

*The attitude mode data used in this paper were taken from housekeeping data; hence, they have a lower time resolution than specified in Brown et al. (2014)

no protuberant parts at launch) and total mass $(\lesssim 4 \mathrm{~kg}$ for 3U) (e.g. Selva and Krejci, 2012). Furthermore, the dimensions restrict the amount of available power from solar cells to $\lesssim 2 \mathrm{~W}$ per unit (e.g. Bouwmeester and Guo, 2010). In terms of magnetic field measurements, typical fluxgate magnetometer instruments used for space plasma physics applications (e.g. Balogh et al., 1997) are thus unsuitable for use on CubeSats since they exceed all of these constraints. Additionally, a full magnetic cleanliness program (e.g. Ludlam et al., 2008) is not possible with CubeSats; thus, the raw data will be contaminated to some degree with fields of spacecraft origin. Therefore, in designing magnetometers (or indeed any scientific instrument) for CubeSat platforms, there must be a trade-off in mass, power and/or precision levels which will affect the instruments' capabilities.

Magnetometers flown on CubeSats thus far have typically been used for attitude purposes (e.g. Sarda et al., 2010). However, there may also be potential science applications for magnetometers on such spacecraft: QuakeSat's single-axis search-coil AC magnetometer has detected lightning-generated whistler mode waves $(10-1000 \mathrm{~Hz})$ and ELF bursts $(10-150 \mathrm{~Hz})$, simultaneously observed on the ground, which were possibly due to earthquakes (Bleier and Dunson, 2005); and DICE's (Dynamic Ionosphere CubeSat Experiment) DC vector magnetometer has detected $\sim 200 \mathrm{nT}$ magnetic deflections due to field-aligned currents at the auroral oval during a marginally geomagnetically active period (Fish et al., 2014). The scientific capabilities that such lowerquality sensors (necessitated by the constraints of CubeSats) offer are as yet not entirely clear. In this paper we assess one such example from the first CINEMA (CubeSat for Ions, Neutrals, Electrons and MAgnetic fields) spacecraft.

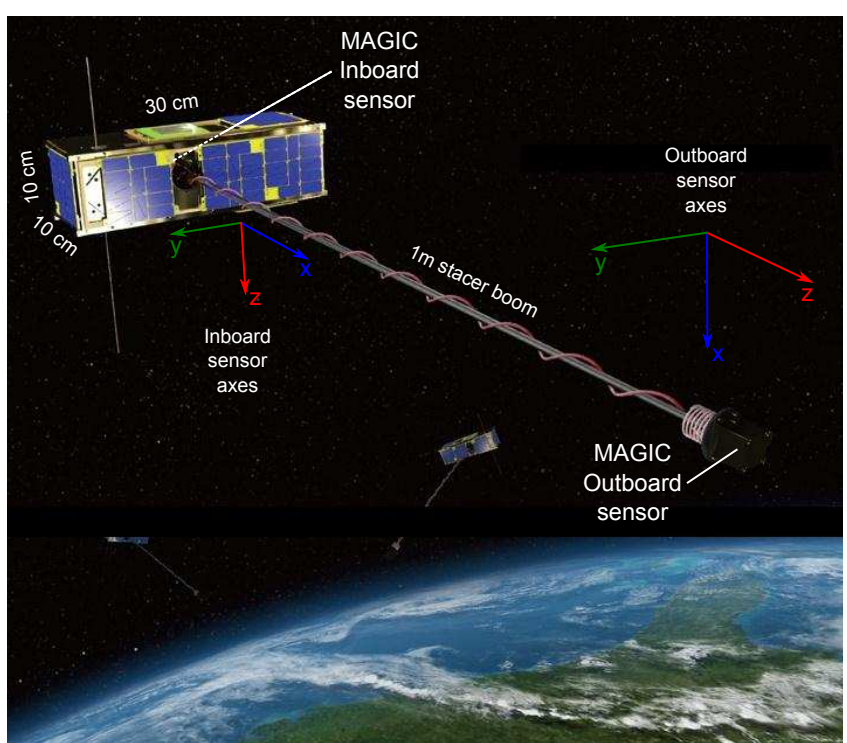

Figure 1. Schematic of CINEMA indicating the inboard (IB) and outboard (OB) MAGIC sensors and their respective axes. Image credit: CINEMA consortium.

CINEMA is a $3 \mathrm{U}$ CubeSat equipped with avionics and science instruments (Vega et al., 2009) launched into low Earth orbit (LEO) on 13 September 2012, with orbital elements shown in Table 1, as a secondary payload from a $\mathrm{P}$ POD (Poly Picosat Orbital Deployer) dispenser. Two additional near-identical CINEMA CubeSats were launched on 3 November 2013 which we do not discuss in this paper. The spacecraft's science instrumentation includes MAGIC (MAGnetometer from Imperial College), two novel miniaturised vector DC magnetometers using anisotropic magnetoresistive (AMR) sensors (Brown et al., 2012, 2014). One sensor, the inboard (IB), is contained within the spacecraft, whereas the other, the outboard (OB), is on the end of a $1 \mathrm{~m}$ stacer boom in order to reduce the effect of spacecraft fields on the measurements. The two sensors and their relative axes are illustrated in Fig. 1. Brown et al. (2014) provide a summary of the modes of operation of the instrument. The requirements of the MAGIC instrument are twofold. Firstly, the sensors (in particular the inboard) should provide measurements of Earth's magnetic field at a level of accuracy suitable for attitude-determination purposes (Vega et al., 2009). Secondly, the outboard sensor should be capable of detecting transient science signals in addition to Earth's field, e.g. magnetic perturbations associated with magnetospheric current systems, important for space weather monitoring (cf. Clausen et al., 2012).

Unfortunately, there have been a number of problems with the spacecraft's systems hence only a limited amount of data has been retrieved from the first CINEMA spacecraft. In this paper we present the first in-flight MAGIC results from the two longest time intervals of MAGIC data obtained for which the onboard clock was reliable. In Sect. 2 we describe the 
attitude-independent calibration procedure used on the raw data, through the use of the International Geomagnetic Reference Field (IGRF). Following calibration, the attitude of the sensors is estimated using a simple magnetometer-only method as described in Sect. 3. Finally, Sect. 4 discusses the small-amplitude $(\sim 20-60 \mathrm{nT})$, transient $(>21 \mathrm{mHz})$ science signals detected by MAGIC in science mode. These are revealed to be field-aligned currents at the auroral oval, which are corroborated by measurements from the DMSP (Defense Meteorological Satellite Program) and POES (Polar-orbiting Operational Environmental Satellites) spacecraft. We, therefore, assess the science capabilities of the MAGIC sensors flown on CINEMA through the use of simple magnetometeronly methods and discuss the possibilities of utilising sensors similar to MAGIC for science purposes in the future.

\section{Attitude-independent calibration}

\subsection{The calibration problem}

The general calibration problem can be written as follows (e.g. Kepko et al., 1996):

$$
\begin{aligned}
\left(\begin{array}{l}
b_{\mathrm{x}} \\
b_{\mathrm{y}} \\
b_{\mathrm{z}}
\end{array}\right)= & \left(\begin{array}{lll}
g_{\mathrm{x}} \sin \theta_{\mathrm{x}} \cos \phi_{\mathrm{x}} & g_{\mathrm{x}} \sin \theta_{\mathrm{x}} \sin \phi_{\mathrm{x}} & g_{\mathrm{x}} \cos \theta_{\mathrm{x}} \\
g_{\mathrm{y}} \sin \theta_{\mathrm{y}} \cos \phi_{\mathrm{y}} & g_{\mathrm{y}} \sin \theta_{\mathrm{y}} \sin \phi_{\mathrm{y}} & g_{\mathrm{y}} \cos \theta_{\mathrm{y}} \\
g_{\mathrm{z}} \sin \theta_{\mathrm{z}} \cos \phi_{\mathrm{z}} & g_{\mathrm{x}} \sin \theta_{\mathrm{z}} \sin \phi_{\mathrm{z}} & g_{\mathrm{z}} \cos \theta_{\mathrm{z}}
\end{array}\right) \\
& \cdot\left(\begin{array}{l}
B_{\mathrm{x}, \mathrm{sc}} \\
B_{\mathrm{y}, \mathrm{sc}} \\
B_{\mathrm{z}, \mathrm{sc}}
\end{array}\right)+\left(\begin{array}{l}
O_{\mathrm{x}} \\
O_{\mathrm{y}} \\
O_{\mathrm{z}}
\end{array}\right),
\end{aligned}
$$

where $\boldsymbol{b}$ consists of the measured magnetic field components from the sensors and $\boldsymbol{B}_{\mathrm{sc}}$ are the real magnetic field vectors in orthogonal, spacecraft-fixed coordinates. The gains $g$ are the scale factors between the physical magnetic field values and the measured values; measurements are always in volts but conventionally a preliminary scale factor $\left(23000 \mathrm{nT} \mathrm{V}^{-1}\right.$, here corresponding to an instrument range of $\pm 57500 \mathrm{nT}$; cf. Brown et al., 2014) is applied so that the gains are of order unity and dimensionless. The angles $\theta$ and $\phi$ correspond to the orientation of each sensor component. Note that the sensor triad is approximately orthogonal by construction, i.e. $\boldsymbol{\theta} \sim(90,90,0)^{\circ}$ and $\boldsymbol{\phi} \sim(0,90,0)^{\circ}$, but in-flight calibration can often determine orientation to better than $0.1^{\circ}$, i.e. better than the triad can be constructed on the ground; hence, non-orthogonality must be allowed for in the calibration process. Finally, the offsets $O$ are systematic errors in the measured fields either inherent to the sensor or due to spacecraft fields. The calibration parameters are, however, not constant over time and will drift depending on the quality of the sensor and the environment it inhabits, e.g. the Cluster fluxgate magnetometers have been found to be remarkably stable with long-term offset drifts of $0.2 \mathrm{nT}$ per year and a temperature dependence of $0.2 \mathrm{nT}^{\circ} \mathrm{C}^{-1}$ (Alconel et al., 2014).

\subsection{Method}

While an initial determination of calibration parameters is usually performed on the ground before launch, unfortunately this was not done for either the inboard or outboard MAGIC sensors that were flown on CINEMA-1. Therefore, the only calibration was determined in-flight, as detailed here. AMR sensors cannot achieve the ultra-high precision and stability of higher-quality magnetometers such as fluxgates; indeed, LEO spacecraft often utilise multiple sensors of different measurement types and capabilities in order to achieve the required precision (e.g. Olsen et al., 2003). Consequently, we aim for a calibration of sufficient quality that spin tone and spacecraft-generated fields do not significantly affect the requirements of the MAGIC instrument, i.e. the ability to determine spacecraft attitude and detect transient physical signals.

Most space plasma scientific spacecraft are spin stabilised, and spectral methods are applied to determine calibration parameters (Kepko et al., 1996), even when the physical field is not known since the incorrect determination of the calibration parameters results in residual spin tones in the despun data. However, in LEO the magnetic field changes rapidly due to the spacecraft motion $\left(\sim 50-90 \mathrm{nT} \mathrm{s}^{-1}\right.$ in CINEMA's orbit); hence, the assumption in this method of a constant field over a spin period does not apply. Furthermore, since the spacecraft's attitude is to be determined from the magnetometer data (see Sect. 3), we must in the first instance use an attitude-independent method of calibration (e.g. Foster and Elkaim, 2008; Springmann and Cutler, 2012). Such methods rely on knowledge of the magnitude of the expected geomagnetic field at the spacecraft location.

We determine the spacecraft position at each time from a two-line element (TLE) set using the SGP4 orbit propagator (Hoots et al., 2004; Vallado et al., 2006). The average time difference from the TLE epoch (the time at which the orbital parameters are referenced), $\Delta t_{\mathrm{TLE}}$ is noted in Table 1. The use of the propagator thus requires the onboard clock to be well calibrated, a factor which limited the number of obtained data intervals from MAGIC which could be used. From the spacecraft positions we calculate the expected field from IGRF $\mathcal{B}$. This model of Earth's inherent magnetic field is accurate to around $5 \mathrm{nT}$ at LEO on average (Maus et al., 2005). However, since IGRF does not include contributions to the magnetic field from magnetospheric current systems, calibration parameters should strictly be determined during geomagnetically quiet times. This was the case for the two intervals used in this paper, as shown in Table 1.

All MAGIC datapoints out of the range of the instrument and large-amplitude spikes were removed before calibration. The attitude-independent calibration procedure used is an iterative procedure. First an initial guess of the (assumed constant) offsets, gains and angles is made. Equation (1) is then inverted at each time $t^{i}$, yielding estimates of the calibrated magnetic field vectors in spacecraft-fixed coordinates $\boldsymbol{B}_{\mathrm{sc}}^{i}$. 


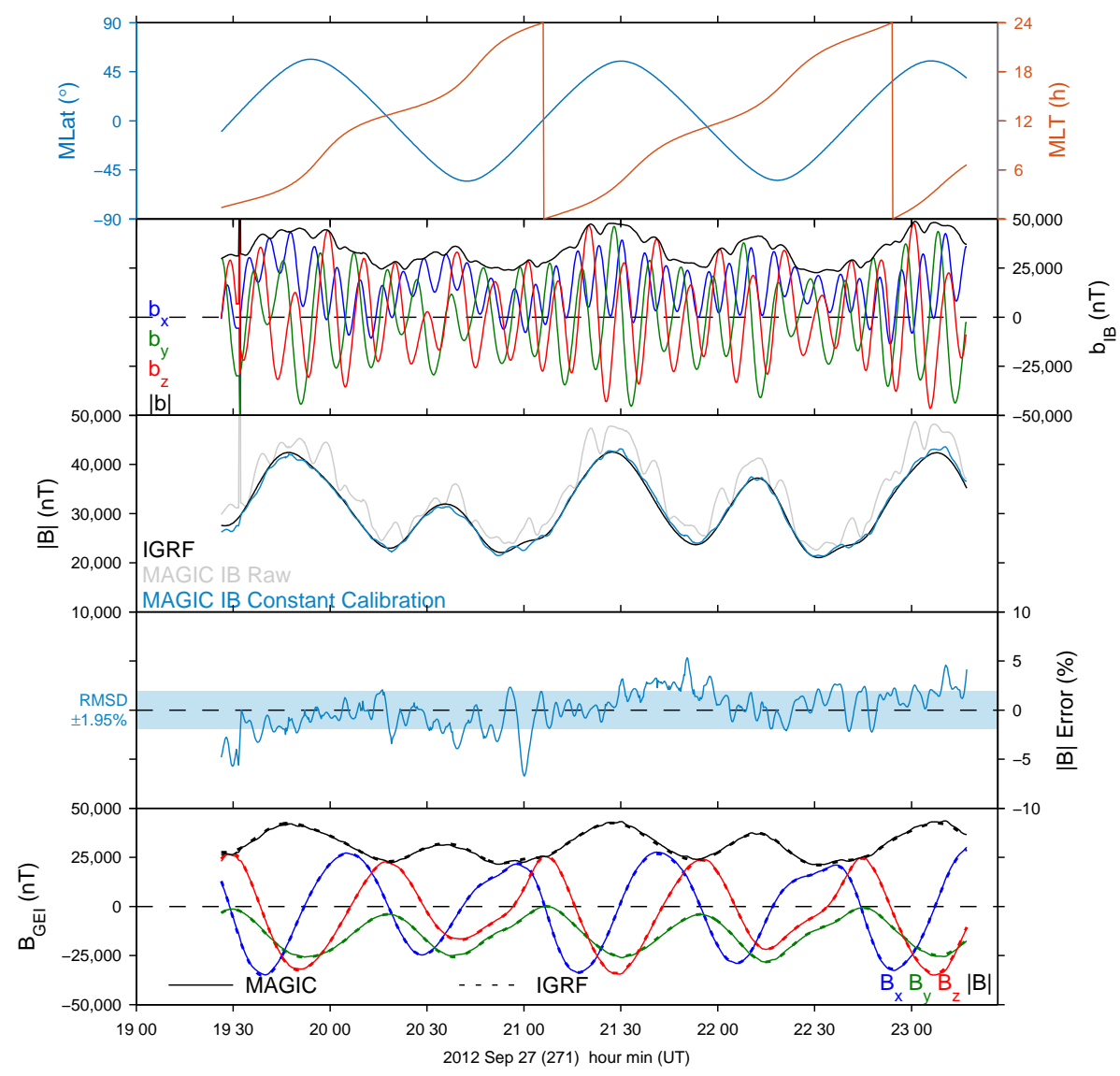

Figure 2. Attitude mode data from the inboard MAGIC sensors. From top to bottom: magnetic latitude (blue) and magnetic local time (orange) of CINEMA; raw data from the three sensors ( $\mathrm{x}, \mathrm{y}, \mathrm{z}$ in blue, green, red) with field strength shown in black; comparison of the raw (grey) and calibrated (blue) data to IGRF (black); percentage error of the calibrated field strength to IGRF, where the shaded area indicates the root mean squared error; comparison of despun calibrated data (solid) with IGRF (dotted) in GEI coordinates.

The square difference in field magnitude from IGRF is then calculated as

$\epsilon=\sum_{i=1}^{N}\left(\left|\boldsymbol{B}_{\mathrm{sc}}^{i}\right|-\left|\mathcal{B}^{i}\right|\right)^{2}$,

where $N$ is the number of datapoints. This algorithm is then iterated in order to minimise $\epsilon$, using the Nelder and Mead (1965) method to obtain successive estimates for the calibration parameters. This is repeated until stable solutions $(\leq 0.01 \%)$ are obtained, a process which typically takes $\sim 1500$ iterations.

\subsection{Results}

\subsubsection{Attitude mode}

Raw attitude mode data from the inboard MAGIC sensor are shown in the second panel of Fig. 2, with a comparison of the measured field magnitude (grey) and IGRF given in the third panel. We despiked the 10-16 s cadence data by removing any datapoints which differed from the previous by more than $10000 \mathrm{nT}$. While the uncalibrated data showed similar variations to IGRF over long timescales, there are shorter timescale oscillatory variations in the data due to the undetermined calibration parameters. Furthermore, MAGIC generally overestimated the field strength in the raw data. We applied the attitude-independent calibration procedure to the data, with the determined calibration parameters displayed in the first row of Table 2.

In order to reliably extract calibration parameters from attitude-independent procedures, the data must have good coverage of the attitude sphere, given by the components of calibrated data normalised by the field magnitude (Foster and Elkaim, 2008). We estimate the data coverage by binning the attitude sphere into 192 equal area bins (cylindrical projection), finding that $69 \%$ of these contained datapoints. Furthermore, we use a $\chi^{2}$ test for complete spatial randomness to quantify the clustering of the data on the attitude sphere, finding $\chi^{2} \sim 4 \chi_{0.025}^{2}$, where $\chi_{0.025}^{2}$ corresponds to the upper limit of the $95 \%$ confidence interval for a Poisson distribution hypothesis. We therefore deduce that, while there was 


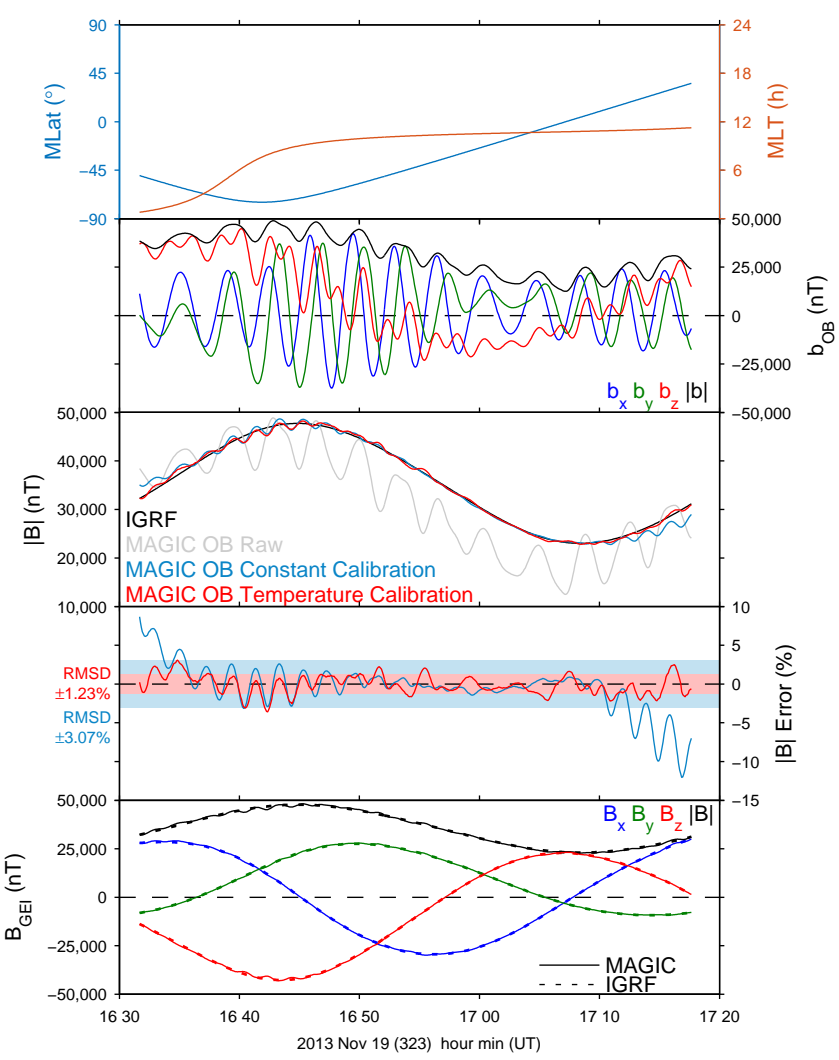

Figure 3. Science mode data from the outboard MAGIC sensors in the same format as Fig. 2. In the third and fourth panels, the red lines correspond to the temperature-dependent calibration.

some clustering, there was fair coverage of the attitude sphere over this interval.

The resulting calibrated magnetic field strength is shown in blue in Fig. 2 (third panel), with the percentage error displayed in the fourth panel. The root mean squared deviation (RMSD) from IGRF of the calibrated attitude mode data was $1.95 \%$ over this interval. These differences are likely due to drifting or time-varying offsets and gains not captured by our constant calibration procedure since the differences (fourth panel) are oscillatory and close to the periods (and harmonics thereof) of the oscillations seen in the raw data (second panel). Nonetheless, the level of accuracy in the absolute field is sufficient for attitude determination, as we demonstrate in Sect. 3. The despun attitude mode data are shown in the bottom panel of Fig. 2.

\subsubsection{Science mode}

Science mode data from the outboard MAGIC sensor are shown in Fig. 3, in the same format as before. Again, before calibration we removed datapoints out of range and despiked the $128 \pm 4 \mathrm{~ms}$ resolution data using a threshold difference of $500 \mathrm{nT}$. It is immediately clear from oscillations in $|\boldsymbol{b}|$ that the offsets were larger for this interval than for the attitude mode data. Furthermore, while the inboard sensor overestimated the geomagnetic field, the outboard generally underestimated it. We applied the attitude-independent calibration procedure only to the first datapoint of each packet ( $5 \mathrm{~s}$ cadence) since these are the datapoints for which times are given (all other times were interpolated), resulting in the parameters listed in the second row of Table 2. Indeed the determined offsets and gains agree with our initial hypothesis in comparison to the attitude mode data. The offsets (which include DC fields of spacecraft origin) for this early development sensor are much larger (by at least a factor of 2) than those determined on the ground for subsequent further-developed AMR sensors (Brown et al., 2014), whereas the gains are within the expected range.

The constant calibration parameters for the science mode data yield an RMSD from IGRF of $3.07 \%$. While this error is in part oscillatory, as with the attitude mode data, the field strength is significantly overestimated at the start of the interval and underestimated at the end. It is known that AMRs have a high dependency on temperature compared to fluxgates (Brown et al., 2014); therefore, a thermistor was packaged with the outboard sensor so that temperature effects could be taken into account. The top panel of Fig. 4 indeed shows that the temperature of the sensor varied a lot over this interval, rising from around $70^{\circ} \mathrm{C}$ at the start to just under $100^{\circ} \mathrm{C}$ at the end, with some small oscillations also at similar periods to those seen in the magnetometer data. The large temperature variations are likely due to the sensor's low thermal inertia, since it was not potted, as well as the fact that CINEMA had been in direct sunlight for $\sim 3$ days prior to this interval.

While the temperature dependence of all the calibration parameters for a sensor would ideally be determined on the ground before launch, Brown et al. (2014) showed that the offsets and gains of MAGIC AMR sensors have an approximately linear relationship with temperature, and Fish et al. (2014) used a linear temperature relationship in their AMR ground calibration. Therefore, we subsequently applied a temperature-dependent calibration to the science mode data to account for the large temperature drift during this interval. This was achieved by modifying the attitude-independent procedure, requiring a linear relationship of the offsets and gains with the temperature measured by the thermistor at each time, e.g. $O_{\mathrm{x}}(t)=c_{\mathrm{x}} T(t)+d_{\mathrm{x}}$, where $O_{\mathrm{x}}(t)$ is now a time-varying magnetometer offset, $T(t)$ is the temperature measured by the thermistor and $c_{\mathrm{x}}$ and $d_{\mathrm{x}}$ are the constants estimated through the iterative calibration procedure. The overall calibration parameters ( $\mathrm{raw} \rightarrow$ temperature calibrated) are listed in the third row of Table 2 and are shown as a function of time in the bottom two panels of Fig. 4. The gains have little temperature dependence and are extremely similar for all three sensor axes. The offsets, on the other hand, show a larger dependence on the temperature (particularly in one component), more so than that determined for later developed sensors which were potted with epoxy resin 
Table 2. List of determined calibration parameters. For temperature calibration, $T$ is in ${ }^{\circ} \mathrm{C}$.

\begin{tabular}{lccc|cccc|rrrrr}
\hline Calibration & \multicolumn{3}{c}{ Gains } & \multicolumn{3}{c|}{ Offsets (nT) } & \multicolumn{4}{c}{ Angles $\left(^{\circ}\right)$} \\
\cline { 2 - 12 } & $g_{\mathrm{x}}$ & $g_{\mathrm{y}}$ & $g_{\mathrm{z}}$ & $O_{\mathrm{x}}$ & $O_{\mathrm{y}}$ & $O_{\mathrm{z}}$ & $\Delta \theta_{\mathrm{x}}$ & $\Delta \theta_{\mathrm{y}}$ & $\Delta \theta_{\mathrm{z}}$ & $\Delta \phi_{\mathrm{x}}$ & $\Delta \phi_{\mathrm{y}}$ & $\Delta \phi_{\mathrm{z}}$ \\
\hline Attitude mode & 1.046 & 1.125 & 1.161 & -673 & 309 & 2082 & 1.07 & -0.43 & -0.01 & -0.01 & 0.31 & -0.00 \\
Science mode & 0.872 & 0.830 & 0.898 & 3488 & 2879 & 6582 & -1.08 & -0.34 & -0.00 & 0.00 & -0.86 & -0.00 \\
(constant) & & & & & & & & & & & & \\
Science mode & $-0.002 T$ & $-0.003 T$ & $-0.003 T$ & $-7.834 T$ & $18.763 T$ & $-155.150 T$ & -1.08 & -0.34 & -0.00 & 0.00 & -0.86 & -0.00 \\
(temperature) & +1.131 & +1.111 & +1.188 & +4185 & +1208 & +20395 & & & & & \\
\hline
\end{tabular}

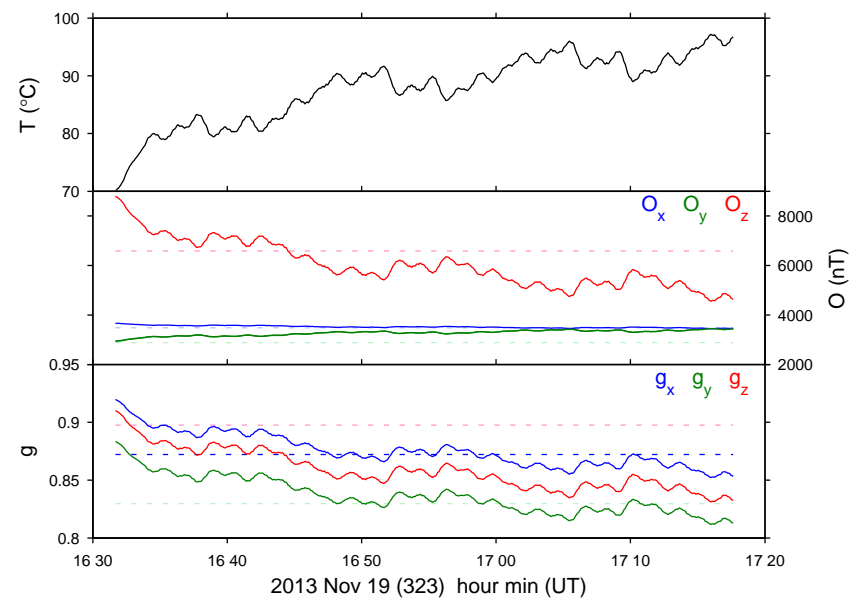

Figure 4. From top to bottom: temperature at the outboard sensors; determined temperature-dependent offsets and gains ( $x, y, z$ in blue, green, red), where the dotted lines indicate the previous constant calibration parameters.

to increase the thermal inertia of the sensors (Brown et al., 2014).

The temperature calibration removes the over- and underestimation of the field at the start and end of the interval respectively and also reduces the amplitude of oscillating deviations, as shown in red on the third and fourth panels of Fig. 3. This calibration results in an RMSD from IGRF of $1.23 \%$, indicated by the red area (Fig. 3, fourth panel), which is just over 1.5 times more accurate than the inboard sensor in attitude mode. In this paper we perform no further calibration on the science mode data; therefore, we treat this RMSD as the absolute accuracy of the outboard MAGIC sensor in science mode. The data covered $85 \%$ of the attitude sphere (not shown) with less clustering than before $\left(\chi^{2} \sim 2 \chi_{0.025}^{2}\right)$; thus, the calibration parameters are likely reliable. Again, we present the despun science mode data, using the method described in Sect. 3, in the final panel of Fig. 3.

\section{Attitude determination}

Following the attitude-independent calibration of MAGIC, we wish to use the magnetometer data to estimate the spacecraft and sensor attitude at each datapoint.

\subsection{Method}

Upon deployment the spacecraft would have been randomly tumbling in its orbit. Whilst an attitude control system was developed for CINEMA utilising magnetorquers (Vega et al., 2009), unfortunately one of the torque coils was not operational, meaning that CINEMA did not successfully detumble. A common method of spacecraft attitude determination is through comparing measurements of vector quantities in spacecraft-fixed coordinates to reference vectors, such as IGRF in the case of magnetic fields. To uniquely determine the attitude at any time thus requires (at least) two independent vector measurements (e.g. Wertz, 1978). Had CINEMA successfully detumbled, the sun sensor would have provided a second vector in addition to the magnetic field (Vega et al., 2009). However, since this was not available, we must therefore estimate the spacecraft attitude using the magnetometer data only.

To represent rotations we use unit quaternions $q=\left\langle\cos \frac{\Theta}{2}\right.$, $\left.\sin \frac{\Theta}{2} \hat{\boldsymbol{w}}\right\rangle$, where $\hat{\boldsymbol{w}}$ is the axis of rotation about which a rotation of $\Theta$ is applied. The rotation from the (calibrated) measured field $\boldsymbol{B}_{\mathrm{sc}}$ in orthogonal, spacecraft-fixed coordinates to IGRF $\mathcal{B}$ in the GEI frame at time $t^{i}$ is given by

$\left\langle 0, \mathcal{B}^{i}\right\rangle=q_{i}\left\langle 0, \boldsymbol{B}_{\mathrm{sc}}^{i}\right\rangle q_{i}^{*}$,

where $q^{*}$ is the conjugate quaternion. We know the family of possible solutions at each time

$q_{i}(\Phi)=\left\langle\cos \frac{\Phi}{2}, \sin \frac{\Phi}{2} \frac{\mathcal{B}^{i}}{\left|\mathcal{B}^{i}\right|}\right\rangle\left\langle\cos \frac{\Theta}{2}, \sin \frac{\Theta}{2} \frac{\boldsymbol{B}_{\mathrm{sc}}^{i} \times \mathcal{B}^{i}}{\left|\boldsymbol{B}_{\mathrm{sc}}^{i} \times \mathcal{B}^{i}\right|}\right\rangle$,

$\cos \Theta=\frac{\boldsymbol{B}_{\mathrm{sc}}^{i} \cdot \mathcal{B}^{i}}{\left|\boldsymbol{B}_{\mathrm{sc}}^{i}\right|\left|\mathcal{B}^{i}\right|}$,

which corresponds to firstly a rotation from the observed to expected field, followed by some arbitrary rotation about the expected field by $\Phi$. Inverting Eq. (3) and taking the time derivative (indicated here by dots), gives

$\left\langle 0, \dot{\boldsymbol{B}}_{\mathrm{sc}}^{i}\right\rangle=\dot{q}_{i}^{*}\left\langle 0, \mathcal{B}^{i}\right\rangle q_{i}+q_{i}^{*}\left\langle 0, \dot{\mathcal{B}}^{i}\right\rangle q_{i}+q_{i}^{*}\left\langle 0, \mathcal{B}^{i}\right\rangle \dot{q}_{i}$,

that is changes in the measured magnetic field can be due to changes in the spacecraft's attitude, i.e. rotation, or due to the 


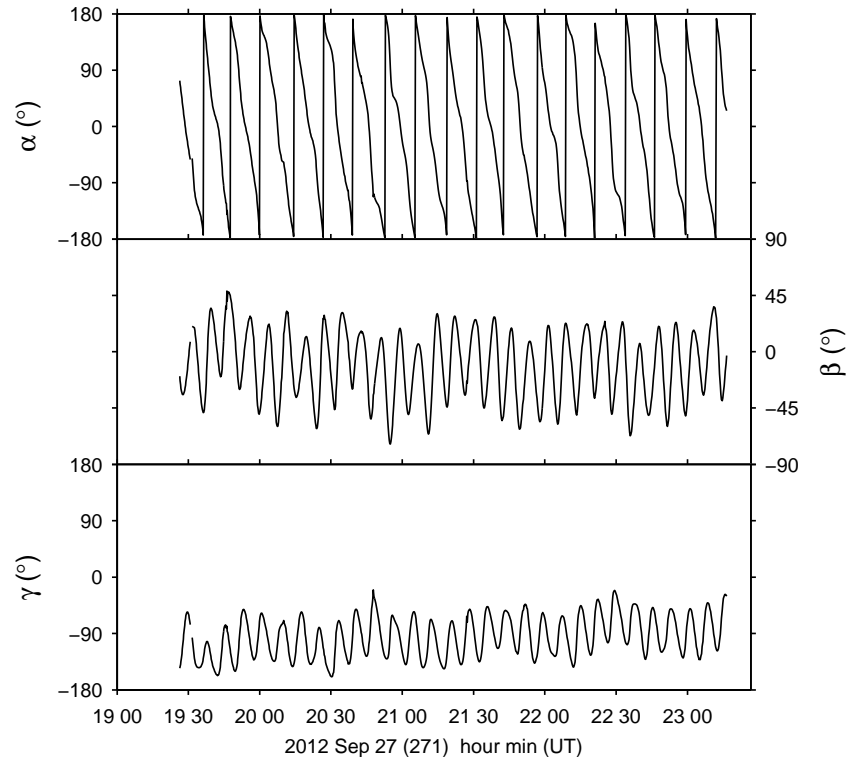

Figure 5. Determined attitude of the inboard MAGIC sensors, represented as the three Euler angles.

real field changing, i.e. through spacecraft motion. In LEO the latter is significant, at $\sim 50-90 \mathrm{nT} \mathrm{s}^{-1}$ for CINEMA.

It is clear from the data that CINEMA was spinning slowly; for example, in the attitude mode data (second panel of Fig. 2), there were $\sim 10$ oscillations of the magnetic field over an entire orbit. Given the cadence of the magnetometer data, the attitude of the spacecraft should thus have only changed by a few degrees at most between each datapoint. We therefore implement a simple method of attitude estimation here, choosing the attitude quaternion $q_{i}(\Phi)$ which best fitted the next datapoint, i.e. the one which minimised the angle between $q_{i}(\Phi)\left\langle 0, \boldsymbol{B}_{\mathrm{sc}}^{i+1}\right\rangle q_{i}^{*}(\Phi)$ and $\mathcal{B}^{i+1}$. This method thus results in attitude estimates at each datapoint, accurate to a few degrees (cf. Natanson et al., 1990).

\subsection{Results}

\subsubsection{Attitude mode}

Figure 5 shows the estimated attitude of CINEMA using the described method, represented as the three Euler angles

$q=\left\langle\cos \frac{\gamma}{2}, \sin \frac{\gamma}{2} \hat{z}\right\rangle\left\langle\cos \frac{\beta}{2}, \sin \frac{\beta}{2} \hat{\boldsymbol{y}}\right\rangle\left\langle\cos \frac{\alpha}{2}, \sin \frac{\alpha}{2} \hat{\boldsymbol{x}}\right\rangle$,

revealing that the spacecraft was spinning about the IB $x$ axis with $\mathrm{a} \sim 12 \mathrm{~min}$ period, along with substantial nutation/precession with a $\sim 8 \mathrm{~min}$ period. This is consistent with the raw data (second panel in Fig. 2), whereby the $y$ and $z$ axes contained the largest oscillations at the spin period with similar amplitudes, whereas the $x$ axis showed much smaller oscillations at a shorter period. Despun attitude mode data are displayed in the bottom panel of Fig. 2.

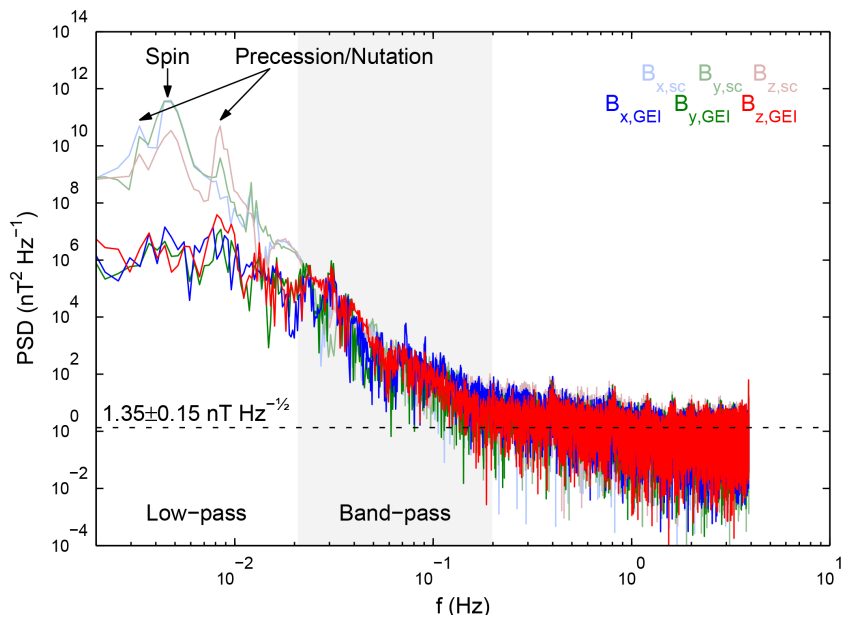

Figure 6. Power spectral densities (PSDs) of the components of the calibrated science mode data (19 November 2013) in both the orthogonal spacecraft fixed frame (lighter) and despun GEI frame, where IGRF has been subtracted from the latter. $x, y$ and $z$ components are given by blue, green and red respectively. The noise level at $1 \mathrm{~Hz}$ in the despun data is indicated by the black dotted line.

This nominal spin axis is along the boom direction (see IB axes in Fig. 1). CINEMA's moment-of-inertia tensor should be largest about the boom axis if it successfully deployed. Therefore, one would expect the spacecraft to spin predominantly about this axis given the initial tumbling out of the P-POD and that one of the torque coils was not operational. Since the magnetometer data show the spacecraft was indeed spinning about the boom axis, we take this as evidence, corroborated by spacecraft onboard systems, that the boom did indeed successfully deploy.

\subsubsection{Science mode}

Before determining the attitude for the science mode data, we applied a low-pass filter using the Morlet wavelet with a cutoff of $21 \mathrm{mHz}$ to remove high-frequency signals and noise. The cutoff was chosen such that spin tones, as shown in Fig. 6, remained. We transform the left-handed sensor axes of the outboard into the same right-handed system as the inboard (see Fig. 1) and subsequently apply the attitude determination procedure every $5 \mathrm{~s}$ to the filtered data. The expected relative orientations of the sensor axes have been corroborated by gradiometer mode data (not shown), whereby data from both sensors are recorded simultaneously (Brown et al., 2014).

The results showed that in the year between the attitude and science mode data in this paper, CINEMA's attitude had substantially changed. This is clear from the power spectra of $\boldsymbol{B}_{\mathrm{sc}}$ in Fig. 6, where there are three different tones (corresponding to spin, precession and nutation) present in all three components. This is unlike the attitude mode data where only two tones were present, one of which was largely confined to 


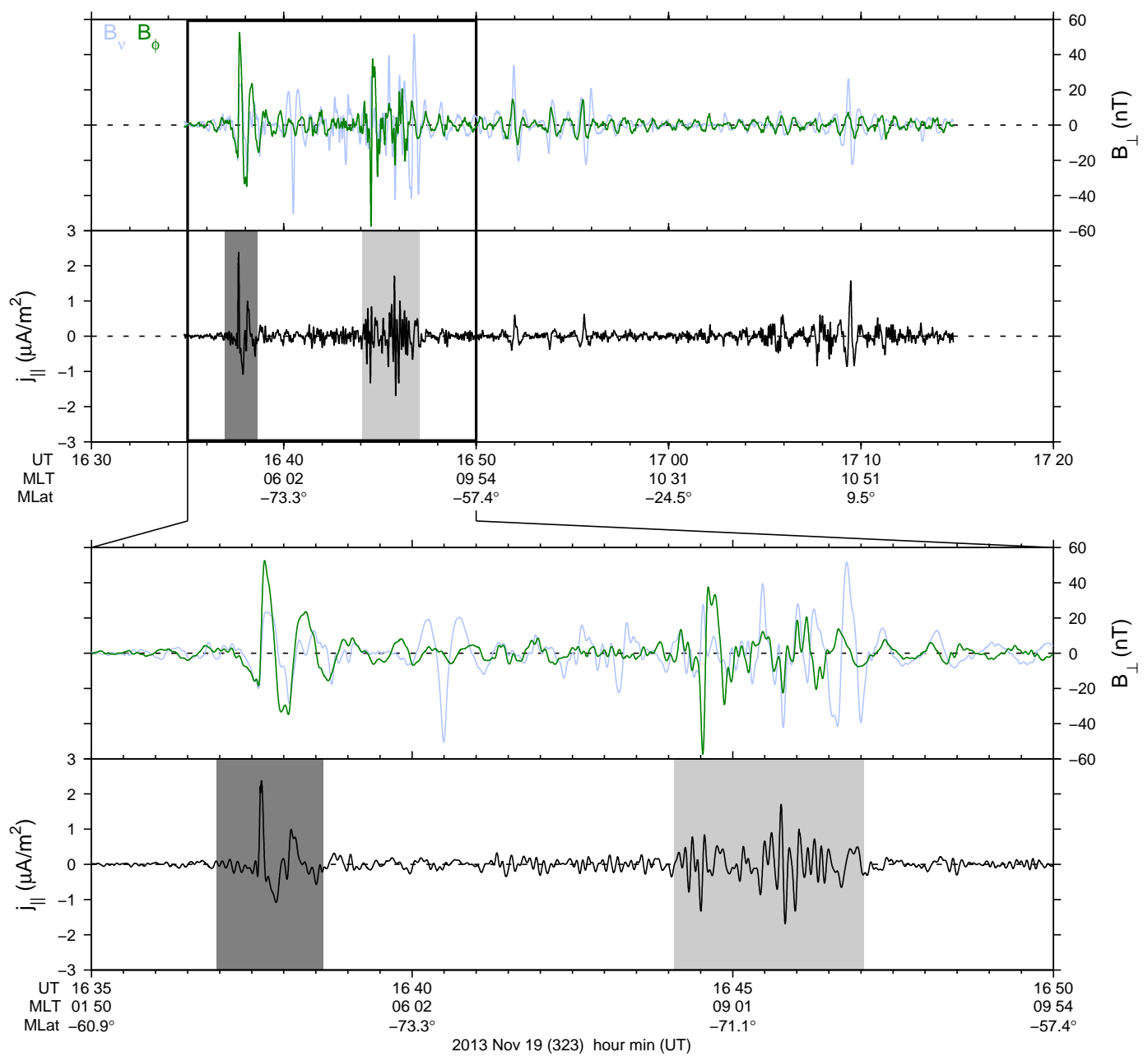

Figure 7. Perpendicular components of the magnetic field (radial in blue, azimuthal in green) and calculated field-aligned currents.

a single axis. The result is that the Euler angles (not shown) are far more complicated than those displayed in Fig. 5.

The despun science mode data are displayed in the bottom panel of Fig. 3. We show power spectra of these components (where IGRF has been subtracted) in Fig. 6, revealing that all three spin tones have been greatly reduced. While errors in the calibration parameters lead to oscillations in the despun data at the spin frequencies, frequencies above the low-pass filter cutoff (in particular in the band-pass region highlighted in Fig. 6) are suitable for science applications, as we demonstrate in the next section.

\section{Field-aligned currents (FACs)}

While we have shown that attitude information can be extracted by comparing the MAGIC data with IGRF, the requirements of the instrument additionally included the ability to detect transient physical signals in the time series due to either spatially or temporally confined phenomena. We transformed the despun MAGIC science mode data in a field- aligned system $(\boldsymbol{v}, \boldsymbol{\phi}, \boldsymbol{\mu})$, where $\boldsymbol{\mu}$ is aligned with IGRF, $\boldsymbol{v}$ is perpendicular to IGRF pointing radially outwards and $\boldsymbol{\phi}$ is the usual azimuthal direction; subsequently, we bandpass filtered the data to reveal transients. A lower cutoff of $21 \mathrm{mHz}$ was used to remove spin tones due to errors in calibration, and the upper cutoff was set at $1.8 \mathrm{~Hz}$ in order to reduce noise and remove quasi-periodic spikes in the data of spacecraft origin.

The two perpendicular components of the magnetic field are shown in Fig. 7, revealing transient signals of $\sim 20$ $60 \mathrm{nT}$ in amplitude, particularly at the start of the interval, when CINEMA was at high magnetic latitudes in the Southern Hemisphere. Through the Ampère-Maxwell law $\mathbf{j}=\nabla \times \boldsymbol{B} / \mu_{0}$, the field-aligned currents (FACs) associated with these magnetic perturbations can be estimated using the method of Lühr et al. (1996), namely

$j_{\|}=\frac{1}{\mu_{0} v_{\perp}} \frac{d}{d t}\left[\boldsymbol{B}_{\perp} \cdot \hat{\boldsymbol{n}}\right]$, 


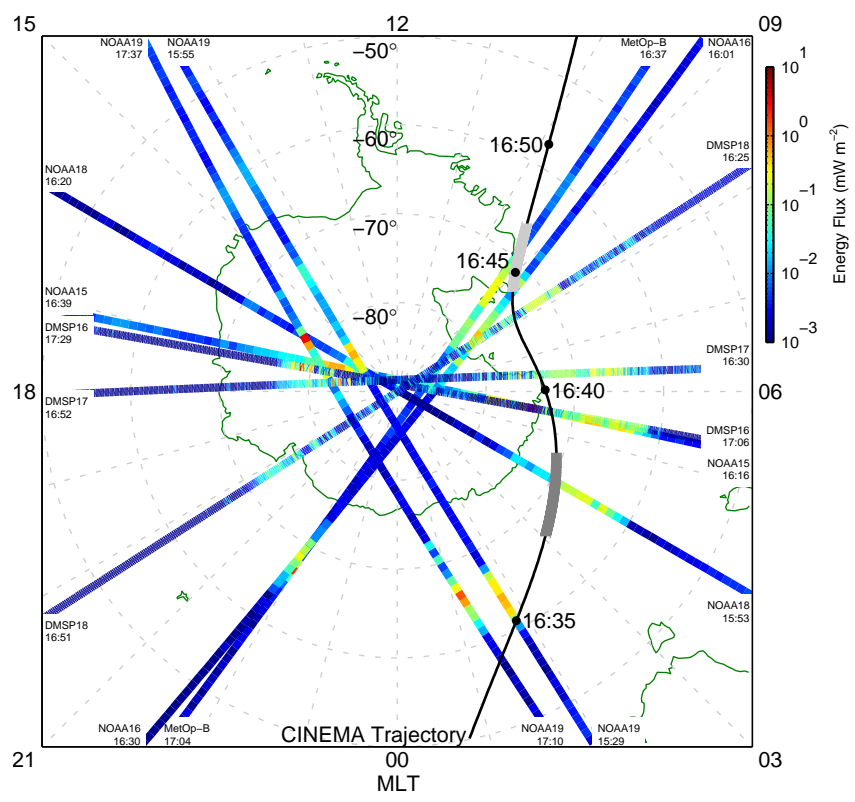

Figure 8. Polar map of the magnetic South Pole in geomagnetic coordinates. CINEMA's trajectory is shown in black, with the two periods of field-aligned currents shown in Fig. 7 highlighted. Total energy fluxes measured by the DMSP (electrons only) and POES (ions and electrons) within $\pm 45 \mathrm{~min}$ of the CINEMA crossing are also shown.

where $v_{\perp}$ is the spacecraft orbital speed perpendicular to IGRF and $\hat{\boldsymbol{n}}=\boldsymbol{\mu} \times \mathbf{v} /|\boldsymbol{\mu} \times \mathbf{v}|$ is a unit vector perpendicular to both IGRF and the orbital velocity. This method can lead to a factor-of- 2 underestimation of the current density due to the finite extent of the (assumed infinite) current sheets (Lühr et al., 1996). The calculated FACs are displayed in the second panel of Fig. 7, showing currents of up to a few $\mu \mathrm{A} \mathrm{m}^{-2}$. We highlight (grey areas) the times of the two periods of FACs between 16:35 and 16:50 UT when CINEMA was traversing the polar cap, where the $S=\overline{\log _{10} j_{\|}^{2}}$ parameter (not shown) of Heilig and Lühr (2013) was used to identify the boundaries. The FACs are qualitatively similar and of similar amplitude to those determined from CHAMP (Challenging Minisatellite Payload) magnetic field data at the auroral oval (Xiong et al., 2014).

To check whether these field-aligned currents are consistent with the location of the auroral oval, we use Total Energy Detector (TED) data from the NOAA POES (Evans and Greer, 2004) and SSJ/5 precipitating particle sensor data from the DMSP spacecraft. Figure 8 displays auroral oval crossings of these spacecraft 45 min either side of the FACs observed by CINEMA, where the tracks have been coloured by the observed total energy fluxes. The POES TED instrument measures energy fluxes into the atmosphere of both ions and electrons in the range $50-20000 \mathrm{eV}$, whereas for DMSP we display only the electron fluxes in the range $30-30000 \mathrm{eV}$ from SSJ/5. CINEMA's trajectory is shown as the black lines, and the two periods of field-aligned currents identified in Fig. 7 are also highlighted. The locations of these FACs are in fairly good agreement with the position of the auroral oval as evidenced from the precipitating particle data; thus, we are confident that MAGIC did indeed detect field-aligned currents at the auroral oval.

A further period of FACs was detected by MAGIC between 17:04:40 and 17:12:20 UT with amplitudes of typically $\sim 0.5 \mu \mathrm{A} \mathrm{m}^{-2}$. During this time CINEMA was near the magnetic equator and only a few degrees eastward of the dawn day-night terminator on the ground. Given this location, we suggest that these could be due to equatorial plasma bubbles, the FAC signatures of which have been detected by CHAMP (Park et al., 2009), revealing similar amplitudes to those presented here. Unfortunately, there is no independent measurement to confirm this interpretation.

\section{Discussion}

In the calibration of the MAGIC data, as presented in Sect. 2, we used an attitude-independent method (e.g. Foster and Elkaim, 2008). In the case of attitude mode, this has assumed constant calibration parameters, whereas for science mode we have added a linear temperature dependence (cf. Brown et al., 2014). Springmann and Cutler (2012) also employed attitude-independent calibration to a commercial offthe-shelf PNI Sensor Corporation MicroMag3 vector magnetometer flown on the RAX-1 (Radio Aurora Explorer) CubeSat in LEO. They found residuals with IGRF of $\sim 900 \mathrm{nT}$, larger than those reported here for MAGIC. However, they subsequently allowed for time-varying biases by modelling (through the Biot-Savart law) telemetered spacecraft currents, reducing the RMSD to $174 \mathrm{nT}$. Such a procedure could be implemented for MAGIC in future flight opportunities as the next step in calibration. Furthermore, following attitude estimation it may be possible to apply attitude-dependent calibration, e.g. by taking into account the induced currents in solar panels due to their illumination.

Our method of attitude estimation (Sect. 3) can be applied to CINEMA only because its tumbling motion is suitably slow. Had CINEMA successfully detumbled and spun up, then the method described here would not have been required since sun sensor data could have been combined with that from MAGIC to uniquely define the spacecraft attitude (Vega et al., 2009). On the other hand, more sophisticated methods of magnetometer-only attitude determination do exist (Natanson et al., 1990; Searcy and Pernicka, 2012). These methods would necessitate further modelling than is possible for CINEMA since they require measures of the spacecraft inertia tensor and any external torques (such as gravity gradients and drags) acting upon it. It is possible that such attitude modelling could be implemented in the future to better constrain spacecraft attitude. 
At present, the determination of physical signals in the MAGIC data (Sect. 4) is limited by a number of factors since both calibration and attitude are all determined through the magnetometer only. The main limiting factor is the period of CINEMA's rotation, precession and nutation. The cutoff in our filtering is chosen such that the low-pass filter will retain these frequencies, whereas the band-pass filter will reduce them. This serves as a limitation on the timescales (corresponding to equivalent length scales here of $\sim 4-360 \mathrm{~km}$ ) of the physical signals which can currently be achieved and could in fact be affecting the determined physical signals and corresponding FACs presented here to some degree. It is possible that a further developed attitude model may reduce these effects.

Both the magnetometer-only calibration and attitude estimation methods used here rely on the real physical magnetic field being, on average, well represented by the International Geomagnetic Reference Field (IGRF) (cf. Maus et al., 2005). While this is certainly the case in low Earth orbit, it is of course not true in general. Nonetheless, AMR sensors similar to MAGIC could be used in other environments, though the methods used to recover accurate magnetic field measurements would have to be tailored to the unique environment and requirements of the instrument.

\section{Conclusions}

We have presented the first in-flight science results from MAGIC (MAGnetometer from Imperial College), a novel miniaturised vector DC magnetometer using anisotropic magnetoresistive (AMR) sensors, aboard the CINEMA (CubeSat for Ions, Neutrals, Electrons and MAgnetic fields) spacecraft in low Earth orbit. We have detailed our attitudeindependent (and temperature-dependent, in the case of science mode) calibration procedures, which result in root mean squared deviations in field magnitude from IGRF of 1.95 and $1.23 \%$ respectively for the inboard (in attitude mode) and outboard (science mode) sensors respectively. Such levels of accuracy in the overall magnetic field are certainly sufficient for attitude estimation (cf. Natanson et al., 1990). Indeed, through the use of magnetometer data only, we estimate CINEMA's attitude to within a few degrees using a simple method, thus characterising the spacecraft's spin, nutation and precession and successfully satisfying the first requirement of the MAGIC instrument.

Furthermore, we have presented evidence that MAGIC is capable of detecting transient physical signals $(\sim 20$ $60 \mathrm{nT}$ ) in addition to simply IGRF, thereby accomplishing the other requirement. These signals were 1 order of magnitude smaller than those detected by the science AMR on the DICE CubeSat during a marginally geomagnetically active period (Fish et al., 2014). Indeed, MAGIC has a resolution and noise floor that are 1 order of magnitude superior to those of the DICE SciMag instrument. The deter- mined field-aligned currents observed by MAGIC $(\sim 0.5-$ $2 \mu \mathrm{A} \mathrm{m}^{-2}$ ) show qualitative agreement with previous observations from the CHAMP spacecraft (Park et al., 2009; Xiong et al., 2014), and those detected at the auroral oval are consistent in location with other available data sets, namely DMSP and POES. Therefore, to our knowledge, MAGIC is the highest-sensitivity vector DC magnetometer flown on a CubeSat to date for which conducting scientific studies is feasible. While AMR sensors cannot achieve the absolute precision of magnetic field measurements at LEO, such as Swarm (Friis-Christensen et al., 2006), certain scientific applications do not require such high levels of precision for which sensors similar to MAGIC could play a role. Indeed we have demonstrated that simple methods applied to only the magnetometer data can yield useful scientific results, such as the locations of field-aligned currents, even during geomagnetically quiet times. The relatively low cost of CubeSats offers the possibility in the future of employing a constellation of spacecraft with MAGIC sensors, e.g. for the purposes of space weather monitoring.

Acknowledgements. We dedicate this paper to Bob Lin of University of California Berkeley, who sadly passed away soon after CINEMA-1's launch. It was his inspiration, drive and enthusiasm which made the CINEMA mission possible. We also thank Alain Hilgers at ESA/ESTEC for support. The development of the hybrid sensor and flight units has been funded by ESA under the General Support Technology Programme (Contract number 4000106430). M. O. Archer is thankful for funding from STFC grant ST/I505713/1. We thank NOAA for POES and DMSP data.

The topical editor E. Roussos thanks L. B. N. C. Clausen and one anonymous referee for help in evaluating this paper.

\section{References}

Acuña, M. H.: Space-based magnetometers, Rev. Sci. Instrum., 73, 3717-3736, doi:10.1063/1.1510570, 2002.

Alconel, L. N. A., Fox, P., Brown, P., Oddy, T. M., Lucel, E. L., and Carr, C. M.: An initial investigation of the long-term trends in the fluxgate magnetometer (FGM) calibration parameters on the four Cluster spacecraft, Geosci. Instrum. Method. Data Syst., 3, 95-109, doi:10.5194/gi-3-95-2014, 2014.

Balogh, A., Dunlop, M. W., Cowley, S. W. H., Southwood, D. J., Thomlinson, J. G., Glassmeier, K.-H., Musmann, G., Lühr, H., Buchert, S., Acuña, M. H., Fairfield, D. H., Slavin, J. A., Riedler, W., Schwingenschuh, K., and Kivelson, M. G.: The Cluster and Phoenix missions, chap. The Cluster magnetic field investigation, Springer Netherlands, 65-91, doi:10.1007/978-94-0115666-0_3, 1997.

Baumjohann, W.: Ionospheric and field-aligned current systems in the auroral zone: a concise review, Adv. Space Res., 2, 55-62, doi:10.1016/0273-1177(82)90363-5, 1982.

Bleier, T. and Dunson, C.: ELF magnetic field monitoring of the San Simeon M6.4 quake from both Quakesat and a ground network, in: Proceedings of the International Workshop on SeismoElectromagnetics, Tokyo, Japan, 15-17 March, 2005. 
Bouwmeester, J. and Guo, J.: Survey of worldwide pico- and nanosatellite missions, distributions and subsytem technology, Acta Astronaut., 67, 854-862, doi:10.1016/j.actaastro.2010.06.004, 2010.

Brown, P., Beek, T., Carr, C., O’Brien, H., Cupido, E., Oddy, T., and Horbury, T. S.: Magnetoresistive magnetometer for space science applications, Meas. Sci. Technol., 23, 025902, doi:10.1088/0957-0233/23/2/025902, 2012.

Brown, P., Whiteside, B. J., Beek, T. J., Fox, P., Horbury, T. S., Oddy, T. M., Archer, M. O., Eastwood, J. P., Sanz-Hernández, D., Sample, J. G., Cupido, E., O’Brien, H., and Carr, C. M.: Space magnetometer based on an anisotropic magnetoresistive hybrid sensor, Rev. Sci. Instrum., 85, 125117, doi:10.1063/1.4904702, 2014.

Clausen, L. B. N., Baker, J. B. H., Ruohoniemi, J. M., Milan, S. E., and Anderson, B. J.: Dynamics of the region 1 Birkeland current oval derived from the Active Magnetosphere and Planetary Electrodynamics Response Experiment (AMPERE), J. Geophys. Res., 117, A06233, doi:10.1029/2012JA017666, 2012.

Evans, D. S. and Greer, M. S.: NOAA Tech. Mem. 1.4, chap. Polar Orbiting Environmental Satellite Space Environment Monitor-2 instrument descriptions and archive data documentation, Space Environment Center, Boulder, CO, USA, 49 pp., 2004.

Fish, C., Swenson, C., Crowley, G., Barjatya, A., Neilsen, T., Gunther, J., Azeem, I., Pilinski, M., Wilder, R., Allen, D., Anderson, M., Bingham, B., Bradford, K., Burr, S., Burt, R., Byers, B., Cook, J., Davis, K., Frazier, C., Grover, S., Hansen, G., Jensen, S., LeBaron, R., Martineau, J., Miller, J., Nelsen, J., Nelson, W., Patterson, P., Stromberg, E., Tran, J., Wassom, S., Weston, C., Whiteley, M., Young, Q., Petersen, J., Schaire, S., Davis, C., Bokaie, M., Fullmer, R., Baktur, R., Sojka, J., and Cousins, M.: Design, Development, Implementation, and On-orbit Performance of the Dynamic Ionosphere CubeSat Experiment Mission, Space Sci. Rev., 181, 61-120, doi:10.1007/s11214-014-0034-x, 2014.

Foster, C. C. and Elkaim, G. H.: Extension of a Two-Step Calibration Methodology to Include Nonorthogonal Sensor Axes, IEEE Aerosp. Electron. Syst. Mag., 44, 1070-1078, doi:10.1109/TAES.2008.4655364, 2008.

Friis-Christensen, E., Lühr, H., and Hulot, G.: Swarm: A constellation to study the Earth's magnetic field, Earth Planets Space, 58, 351-358, 2006.

Heilig, B. and Lühr, H.: New plasmapause model derived from CHAMP field-aligned current signatures, Ann. Geophys., 31, 529-539, doi:10.5194/angeo-31-529-2013, 2013.

Hoots, F. R., Schumacher Jr., P. W., and Glover, R. A.: History of analytical orbit modeling in the U.S. space surveillance system, J. Guid. Control Dynam., 27, 174-185, doi:10.2514/1.9161, 2004.

Kepko, E. L., Khurana, K. K., Kivelson, M. G., Elphic, R. C., and Russell, C. T.: Accurate determination of magnetic field gradients from four point vector measurements - part I: Use of natural constraints on vector data obtained from a single spinning spacecraft, IEEE T. Magn., 32, 377-385, doi:10.1109/20.486522, 1996.

Li, X., Schiller, Q., Blum, L., Califf, S., Zhao, H., Tu, W., Turner, D. L., Gerhardt, D., Palo, S., Kanekal, S., Baker, D. N., Fennell, J., Blake, J. B., Looper, M., Reeves, G. D., and Spence, H.: First results from CSSWE CubeSat: Characteristics of relativistic electrons in the near-Earth environment during the Oc- tober 2012 magnetic storms, J. Geophys. Res., 118, 6489-6499, doi:10.1002/2013JA019342, 2013.

Ludlam, M., Angelopoulos, V., Taylor, E., Snare, R. C., Means, J. D., Ge, Y. S., Narvaez, P., Auster, H. U., Le Contel, O., Larson, D., and Moreau, T.: The THEMIS magnetic cleanliness program, Space Sci. Rev., 141, 171-184, doi:10.1007/s11214-008-9423-3, 2008.

Lühr, H., Warnecke, J. F., and Rother, M. K. A.: An algorithm for estimating field-aligned currents from single spacecraft magnetic field measurements: a diagnostic tool applied to Freja satellite data, IEEE T. Geosci. Remote, 34, 1369-1376, doi:10.1109/36.544560, 1996.

Maus, S., Macmillan, S., Lowes, F., and Bondar, T.: Evaluation of Candidate Geomagnetic Field Models for the 10th Generation of IGRF, Earth Planets Space, 57, 1173-1181, 2005.

Natanson, G. A., McLaughlin, S. F., and Nicklas, R. C.: A method of determining attitude from magnetometer data only, in: Flight Mechanics/Estimation Theory Symposium, NASA Goddard Space Flight Center, 359-378, 1990.

Nelder, J. A. and Mead, R.: A simplex method for function minimization, Computer Journal, 7, 308-313, doi:10.1093/comjnl/7.4.308, 1965.

Olsen, N., Tøffner-Clausen, L., Sabaka, T. J., Brauer, P., Merayo, J. M. G., Jørgensen, J. L., Leger, J.-M., Nielsen, O. V., Primdahl, F., and Risbo, T.: Calibration of the Ørsted vector magnetometer, Earth Planets Space, 55, 11-18, 2003.

Park, J., Lühr, H., Stolle, C., Rother, M., Min, K. W., and Michaelis, I.: The characteristics of field-aligned currents associated with equatorial plasma bubbles as observed by the CHAMP satellite, Ann. Geophys., 27, 2685-2697, doi:10.5194/angeo-27-26852009, 2009.

Sarda, K., Grant, C., Eagleson, S., Kekez, D., and Zee, R.: Canadian Advanced Nanosatellite Experience 2 Orbit Operations: Two Years of Pushing the Nanosatellite Envelope, in: Proceedings of the 61st International Astronautical Congress, 1-13, 2010.

Searcy, J. D. and Pernicka, H. J.: Magnetometer-only attitude determination using novel two-step kalman filter approach, J. Guid. Control Dynam., 35, 1693-1701, doi:10.2514/1.57344, 2012.

Selva, D. and Krejci, D.: A survey and assessment of the capabilities of Cubesats for Earth observation, Acta Astronaut., 74, 50-68, doi:10.1016/j.actaastro.2011.12.014, 2012.

Springmann, J. C. and Cutler, J. W.: Attitude-independent magnetometer calibration with time-varying bias, J. Guid. Control Dynam., 35, 1080-1088, doi:10.2514/1.56726, 2012.

Vallado, D. A., Crawford, P., Hujsak, R., and Kelso, T. S.: Revisiting Spacetrack Report \#3, in: AIAA/AAS Astrodynamics Specialist Conference and Exhibit, AIAA 2006-6753, Keystone, CO, USA 2006.

Vega, K., Auslander, D., and Pankow, D.: Design and modeling of an active attitude control system for cubesat class satellites, in: AIAA Modeling and Simulation Technologies Conference, AIAA 2009-5812, Chicago, Illinois, doi:10.2514/6.2009-5812, 2009.

Wertz, J. R.: Spacecraft attitude determination and control, D. Reidel, Dordrecht, the Netherlands, 1978.

Xiong, C., Lühr, H., Wang, H., and Johnsen, M. G.: Determining the boundaries of the auroral oval from CHAMP fieldaligned current signatures - Part 1, Ann. Geophys., 32, 609-622, doi:10.5194/angeo-32-609-2014, 2014. 